\title{
Escritura académica en tres escuelas de ingeniería chilenas: la familia 'Informe técnico' como macrogénero discursivo en el área de Ingeniería Civil Informática
}

Academic writing in three Chilean engineering schools: The 'technical report' family as a discourse macrogenre in Computer Science Civil Engineering

\author{
Enrique Sologuren Insúa ${ }^{1}$ \\ enrique.sologuren@uchile.cl \\ Universidad de Chile, Chile
}

\section{Resumen:}

Los géneros producidos por estudiantes han comenzado a recibir mayor atención debido a su diversidad de funciones en el aprendizaje disciplinar. Así, este trabajo se enmarca en el ámbito del estudio de géneros de formación en español. El objetivo de esta investigación es identificar, definir y caracterizar los géneros de la familia 'informe técnico' con el objeto de comprender su rol formativo en la enseñanza de la ingeniería civil y en los procesos de alfabetización académica-profesional en esta disciplina. El estudio se aborda desde un enfoque cualitativo, particularmente desde la teoría del género del discurso y explora el macrogénero informe técnico en ingeniería civil informática (MGITEC) en el corpus de aprendientes HÉLICE-2017. Esta familia genérica presenta una alta relevancia en el discurso acadé-

\begin{abstract}
:
Genres written by students have received significant attention due to its diversity of functions in subject learning. Thus, the present article is framed within the study field of academic genres in Spanish. The aim of this research is to identify, define and characterize genres of the 'technical report' family to understand its educational role in civil engineering and in the processes of professional-academic literacy in this field. The study adopts a qualitative approach, specifically from the discourse genre theory and explores the technical report macrogenre in computer science civil engineering (MGITEC, for its acronym in Spanish) in the student corpus HÉLICE-2017. This genre family is highly relevant in the academic discourse of civil engineering and in the subdiscipline com-
\end{abstract}

1 Dirección para correspondencia (correspondence address):

Enrique Sologuren Insua. Av. Beauchef $N^{\circ} 850$, Piso 3, comuna de Santiago, Santiago de Chile. CP: 8370448 (Chile). 
Escritura académica en tres escuelas de ingeniería chilenas: la familia 'Informe técnico' como macrogénero discursivo en el área de Ingeniería Civil Informática

EnRIQUe SOlOGURen Insúa

mico de la ingeniería civil y en la subdisciplina informática. Los resultados del análisis de los corpus textuales evidencian la gran diversidad de recursos genéricos utilizados en la comunidad de práctica concernida. A partir de los datos obtenidos se identificó un continuum de los géneros discursivos que van desde informes orientados a la inserción académica hasta la producción escrita de informes orientados a contextos profesionales. En él se despliega una amplia variedad de tipos de informes que cumplen propósitos formativos de relevancia en la aplicación de pensamiento analítico, crítico y creativo para la resolución de problemas: la raison d'être de la ingeniería. Finalmente, la investigación permite configurar relaciones intergenéricas en la escritura académica de informes y proyectar estrategias didácticas de alfabetización disciplinar en el ámbito de la ingeniería.

\section{Palabras clave:}

Escritura; ingeniería; corpus para el aprendizaje; macrogénero informe técnico. puter science. Results of the text corpus analysis reveal a great diversity of genre resources used in this community of practice. In consequence, a continuum of discourse genres was identified based on the collected data, from reports of academic insertion to reports of professional context. Different types of reports are displayed in this continuum. These reports meet relevant educational purposes when it comes to using analytical, critical and creative thinking for problem solving: the raison $d^{\prime}$ être of engineering. Finally, this piece of research makes it possible to define relations between genres in the academic writing of reports and to provide didactic strategies for subject literacy in engineering.

\section{Key words:}

Writing; engineering; learner corpus; technical report macrogenre.

\section{Résumé:}

Les genres produits par les étudiants ont commencé à faire l'objet d'une plus grande attention en raison de la diversité de leurs fonctions dans l'apprentissage disciplinaire. Ainsi, ce travail s'inscrit dans le cadre de l'étude des genres de formation en espagnol. L'objectif de cette recherche est d'identifier, de définir et de caractériser les genres de la famille des "rapports techniques", afin de comprendre leur rôle formateur dans l'enseignement du génie civil et dans les processus d'alphabétisation universitaire dans cette discipline. L'étude est abordée selon une approche qualitative, notamment à partir de la théorie du discours sur le genre, et explore le macro-genre qui est le rapport technique en génie informatique civil (MGITEC) dans le corpus des apprenants d'HÉLICE-2017. Cette famille générique a une grande importance dans le discours académique du génie civil et dans la sous-discipline de l'informatique. Les résultats de l'analyse des corpus textuels montrent la grande diversité des ressources génériques utilisées dans la communauté du stage pratique concerné. À partir des données obtenues, un continuum de genres discursifs a été identifié, allant des rapports orientés vers l'insertion académique à la production écrite de rapports orientés vers des contextes professionnels. Cette dernière présente une grande variété de types de rapports qui, de façon notable, servent à des fins de formation dans l'application de la pensée analytique, critique et créative pour la résolution de problèmes : la raison d'être de l'ingénierie. Enfin, la recherche permet de configurer les relations inter-génériques dans la rédaction académique des comptes-rendus et de projeter des stratégies didactiques d'alphabétisation disciplinaire dans le domaine de l'ingénierie.

\section{Mots clés:}

Rédaction; ingénierie; corpus d'apprentissage; compte-rendu technique macrogenre.

Fecha de recepción: 22-10-2020

Fecha de aceptación: 27-01-2021 


\section{Introducción}

Escribir en las disciplinas conforma un ámbito de exploración, indagación y enseñanza-aprendizaje que nos convoca a seguir estudiando las prácticas discursivas en diferentes culturas, disciplinas, niveles y tramos educativos, ya que:

"Las prácticas de escritura y lectura (o prácticas letradas, de alfabetismo o
literacidad) se ejercitan en -casi- todos los ámbitos: en la vida privada, en
la arena política, en el trabajo, en la iglesia, etc. En la mayoría de estos ám-
bitos existen instituciones y organismos que ordenan la manera de usar esas
prácticas, con tradiciones, hábitos, normas y modelos que fijan en grados
variados la estructura de los textos, su función, los roles que adoptan sus
interlocutores, el poder que adquieren, etc." (Cassany y López, 2010, p. 347).

En este sentido, ahondar en las prácticas de escritura nos permite comprender de mejor manera la naturaleza de los textos utilizados en la educación superior (Morales, Perdomo, Cassany e Izarra, 2020; Cordero y Carlino, 2019). El objetivo de este artículo es describir la familia genérica 'Informe Técnico' a partir de un corpus textual producido por estudiantes de ingeniería civil informática de tres universidades chilenas; $y$, que es utilizado para acreditar su conocimiento disciplinar a lo largo del plan de estudios. El estudio se aborda desde un enfoque cualitativo (Angouri, 2010), particularmente desde la teoría del género del discurso (Swales, 2004).

En este trabajo se presentan los hallazgos relacionados con la identificación y caracterización de los géneros de formación académica en ingeniería civil informática (GEFIC). Para esto, nos focalizamos en aqueIlos de mayor relevancia cuantitativa y cualitativa a partir del análisis multidimensional del corpus textual HÉLICE 2017. Todos estos géneros son producidos y utilizados en el ciclo formativo terminal de los programas de pregrado analizados.

Esta familia genérica presenta una alta relevancia en el discurso académico de la ingeniería civil informática y en otras subdisciplinas de la ingeniería. Se trata también de un género frecuente en los espacios profesionales junto con "specifications, proposal writing, requirements, and various reports" (Sales, 2002, p. 83).

En lo que sigue se entregan los fundamentos teóricos y metodológicos 
Escritura académica en tres escuelas de ingeniería chilenas: la familia 'Informe técnico' como macrogénero discursivo en el área de Ingeniería Civil Informática

ENRIQUE SOlOgUREN INSÚA

específicos para el análisis de este subcorpus textual, luego, se presentan los resultados sobre la caracterización de este macrogénero o familia de géneros y finalmente, se presentan conclusiones y proyecciones para el ámbito de la alfabetización académica en las ingenierías.

\section{El informe como género y macrogénero del discurso}

El género informe académico ha sido definido como una interacción que cumple un propósito social particular dentro de las variadas prácticas discursivas, utilizadas por los estudiantes en las carreras de pregrado y que permite estimar un nivel de desarrollo en un área disciplinar específica. En él participan un estudiante universitario como enunciador y un académico como destinatario, el que evalúa la aplicación y cumplimiento de convenciones textuales-discursivas (Harvey y Muñoz, 2006). En cuanto a la noción de informe, Harvey (2005) señala que el informe es un tipo de texto de circulación académica cuyas representaciones, según resultados de su investigación, difieren cuando se relevan las funciones que le son atribuidas por profesores y estudiantes. Distinguió cuatro tipos de informes: el bibliográfico, el de diagnóstico, el de caso y el de investigación (IMRD). La función que cumplen es la "transmisión de un contenido que responde a una tarea específica en el caso de los estudiantes, y su posterior evaluación" (Harvey, 2005, p. 218).

Para Amieva un informe puede ser definido como una "exposición escrita relativa a un tema, problema o actividad con propósitos formales de comunicación" (2001, p. 1). En el ámbito de la enseñanza de la ingeniería los informes se posicionan como herramientas habituales en tareas académicas y profesionales para desarrollar competencias laborales como el diseño de situaciones específicas, la implementación de propuestas y el desarrollo de habilidades no técnicas para un desempeño adecuado en la empresa a nivel personal e interpersonal (Amieva, 2001).

Otros estudios se han enmarcado en patrones léxicos (Muñoz, 2006), en la construcción temática y la materialización discursiva en los informes de estudiantes de pregrado (Tapia- Ladino y Burdiles, 2009; Sologuren \& Castillo, 2019), en propuestas sobre cómo evaluarlos desde el punto de vista lingüístico y discursivo (Tapia-Ladino, Burdiles y Arancibia, 2003) y en el análisis de las dificultades y errores frecuentes más frecuentes a la hora de escribirlos (Arechabala, Catoni, Ávila, Riquelme 
y Aedo, 2011). Recientemente Ávila y Cortés (2017) han caracterizado el informe de caso en el área de Educación con una perspectiva de Escribir a través del curriculum.

Nathan (2016) analiza el informe de caso de negocios producido por estudiantes de postgrado nativos y no nativos de inglés en los cursos de marketing y gestión de marketing en una universidad británica. A partir de sus hallazgos configura el modelo retórico discursivo para este género de formación y releva algunas características salientes en la configuración de este recurso genérico, las que se encuentran estrechamente relacionadas con el grado de especialización disciplinar.

Parkinson (2017), con una perspectiva anclada en el análisis del género, ha definido la organización del informe de laboratorio producido por estudiantes universitarios a partir de los datos que provee el corpus BAWE (Gardner y Nesi, 2013). Esta organización retórica presenta algunas diferencias significativas en términos de realización de movidas con los artículos de investigación, dado el especial tipo de relación entre escritor-lector y los propósitos diferentes de cada uno. En especial, cuando el informe de laboratorio presenta una orientación pedagógica. Esto es importante para entender que en el aula universitaria los docentes toman decisiones y promueven formas de escritura que no necesariamente tendrán una correspondencia unívoca con un género experto.

Dentro de la Escuela Lingüística de Valparaíso, el informe es caracterizado por Parodi, Ibáñez, Venegas y González (2010) como un:

Género discursivo cuyo macropropósito es consignar situaciones, procedimientos y/o problemas. Idealmente, su contexto de circulación es el ámbito laboral y la relación entre los participantes es entre escritor experto y lector experto. Suele ser monomodal y presentar un modo de organización discursiva que es descriptiva (p. 276).

Así, el informe se configura como un género propio del ámbito profesional y puede circular también en el ámbito académico, en diferentes contextos de comprensión y producción de textos académicos. Desde la escuela de Sídney, el informe puede ser clasificado como una explicación procedural (Martin y Rose, 2007). También en contextos anglosajones de formación en ingeniería, el género informe se ha constituido en una herramienta de aprendizaje eficaz, en esa línea destaca el trabajo de Walker (2013), quien asume la noción de género para enseñar a estu- 
Escritura académica en tres escuelas de ingeniería chilenas: la familia 'Informe técnico' como macrogénero discursivo en el área de Ingeniería Civil Informática

ENRIQUE SOlOgUREN INSÚA

diantes de ingeniería a escribir informes colaborativamente, integrando la escritura en los propios cursos de la disciplina.

Asimismo, en disciplinas específicas como la economía el informe técnico ha sido reconocido como un macrogénero y abordado desde el estudio del discurso profesional:

El Informe Técnico se constituye como un macrogénero, el cual agrupa a diversos informes provenientes del ámbito profesional que se diferencian, entre otras cosas, por las temáticas que tratan, las instituciones que los emiten, las audiencias originales declaradas, y los grados de especialización en la materia informada (Parodi, Julio y Vásquez-Rocca, 2015, p. 186).

Dentro de este macrogénero o familia entendida como un grupo de géneros similares (Gardner, 2012, p. 56) se ha identificado un conjunto amplio de géneros específicos, para los que actualmente contamos con caracterizaciones detalladas de: El Informe de Política Monetaria (Parodi et al., 2015; Vásquez-Rocca, 2016; Vásquez-Rocca y Parodi, 2015), el Informe de Empleo Regional (Oyarzún, 2016), el Informe Mensual de Estadísticas Monetarias y Financieras (Molina, Vásquez-Rocca y Parodi, 2018), el Informe Anual del Fondo Monetario Internacional (Moreno-de León, 2017), y, el Informe de Estabilidad Financiera (González y Burdiles, 2018), todos géneros especializados de carácter profesional y producidos por expertos en materia económica.

También en el campo del discurso profesional, Montolío (2010) y Montolío y López (2010) analizan informes de consultoría producidos por expertos del ámbito tecnológico y caracterizan la recomendación experta y el asesoramiento profesional como operaciones textuales muy significativas en la producción escrita cotidiana de un número creciente de profesionales. Así mismo junto con caracterizar los mecanismos lingüísticos y discursivos de este género, fuertemente asociado con una organización discursiva argumentativa, releva las dificultades que los profesionales manifiestan para su elaboración.

En el campo de la medicina Da Cunha y Montané (2019) registran el informe técnico como un género que es identificado por profesores y estudiantes del área de medicina y el género informe reportado por estudiantes y profesionales de los estudios de Turismo. Gardner (2012) explora el contexto social de la escritura estudiantil con fines evaluativos en dos familias de géneros que presentan una importante presencia de 
informes: Reporte de investigación y Recuento metodológico. De este modo, a partir del análisis del registro y del género de los textos que presentan una estructura IMRD canónica (Introducción, métodos, resultados y discusión) distingue características lingüísticas desplegadas en diversos contextos disciplinares y en diferentes niveles de estudio. Y, con una importante diversidad de propósitos evaluativos, pedagógicos e internos. Estos últimos, forman parte de los ingredientes que a juicio de Artemeva (2009) contribuyen en la formación del conocimiento genérico profesional.

Se trata de géneros de relevancia para el desarrollo disciplinar especializado de los estudiantes y desde nuestro conocimiento, aún es escasa la información disponible sobre las clases textuales que los estudiantes escriben con propósitos formativos en la educación superior. Asimismo, se hace necesario contar, por un lado, con descripciones basadas en corpus de lo que efectivamente escriben los estudiantes y que consideren sus características retórico-discursivas, ya que a partir de ellas se podría modelar la práctica discursiva y potenciar el aprendizaje de los estudiantes. En esta línea, los análisis realizados sobre relatos de vida lectora de Castillo y Sologuren (2020) dan cuenta no sólo de la riqueza léxica de profesores en formación, sino también del grado de desarrollo de sus habilidades discursivas. Y, por otro lado, con diagnósticos que ayuden a proponer estrategias para la mejora de la escritura académica dentro de la educación superior. En este ámbito se enmarcan los trabajos de Romero, Salvador y Trigo, (2014) realizados en el ámbito de la pedagogía, y Trigo y Núñez, (2018), que analizan los procesos de escritura académica en lengua extranjera.

\section{Corpus y metodología}

El corpus HÉLICE-2017 es un corpus textual (Castillo, 2020) que puede ser catalogado como un corpus de aprendientes o para el aprendizaje (Ferreira, Ejalde y Vine 2014). Este puede ser definido como un conjunto de textos específicos producidos por estudiantes universitarios de una determinada área de estudio, en este caso ingeniería civil en informática. Se trata de un corpus especializado (Gilmore y Millar, 2018) y su construcción está motivada por la hipótesis siguiente: "that this would form a rich source of naturally occurring discourse in support of the students' 
Escritura académica en tres escuelas de ingeniería chilenas: la familia 'Informe técnico' como macrogénero discursivo en el área de Ingeniería Civil Informática

ENRIQUE SOlOgUREN INSÚA

learning, and thus gaining information on the most appropriate way to develop the corpus" (Rodgers, Chambers y Le Baron-Earle, 2011).

En este sentido, con el objetivo de describir los géneros de formación académica producidos por los estudiantes del ciclo terminal de ingeniería civil informática como parte de las exigencias de sus cursos de especialidad se desarrolla este corpus. Este corpus multigéneros contiene 468 textos pertenecientes a tres carreras de Ingeniería civil informática de tres universidades chilenas de reconocido prestigio (Quacquarelli, 2019) y elaborados entre los años 2015 a 2019. El corpus contiene 1.413.437 palabras, cantidad que excede el mínimo de un millón de palabras recomendada para los corpus especializados (Rea Rizzo, 2010). En la tabla siguiente se pueden observar las principales características de las instituciones participantes en el estudio.

Tabla 1

Las cinco mejores universidades de Chile según estándares internacionales (Quacquarelli Symonds, 2019)

\begin{tabular}{llc} 
Universidad & Casa Central & Tipo \\
\hline Universidad de Chile & Santiago & Estatal \\
\hline Pontificia Universidad Católica de Chile & Santiago & Privada \\
\hline Universidad de Concepción & Concepción & Privada \\
\hline Pontificia Universidad Católica de Valparaíso & Valparaiso & Privada \\
\hline Universidad Técnica Federico Santa María & Valparaíso & Privada \\
\hline
\end{tabular}

Así mediante un enfoque ascendente-descendente (Biber, Connor y Upton, 2007; Ibáñez et al., 2017), y utilizando como marco inicial la propuesta de Parodi et al. (2015), estos géneros fueron identificados y posteriormente caracterizados y definidos a partir de un conjunto de criterios tales como propósito comunicativo, organización discursiva, modo semiótico, contexto de circulación, relación entre los participantes y objetivo de aprendizaje.

Para la obtención del corpus se desarrolló un muestreo no probabilístico intencionado (Pagano, 2012), por conveniencia u oportunista (Corbetta, 2007). Dado que se trata de géneros ocultos (Swales, 1996), de 
difícil obtención, se siguió una estrategia de recolección que consistió en solicitar a todos los estudiantes del ciclo formativo terminal o profesional el envío de la mayor cantidad posible de trabajos escritos aprobados $(>=5,5)$ realizados en el marco de los cursos del ciclo terminal o capstone. Por lo que la constitución del corpus emanó de la selección realizada por los mismos estudiantes que rindieron estos cursos y, en ese sentido, presenta algunas características propias de los corpus autocompilados (Lee y Swales, 2006). En total, 103 estudiantes de los tres programas académicos aportaron con textos para la conformación del corpus. En promedio, cada estudiante aportó cinco textos. Los cursos del ciclo terminal de cada una de las carreras se detallan en el anexo $\mathrm{N}^{\circ} 1$.

\section{Resultados y discusión: El informe técnico en ingeniería civil informática}

Para Bhatia (2016) las teorías del género se encuentran permanentemente desafiadas y de diversas formas; por lo que este autor denomina "el mundo real de discurso". En este sentido, los géneros, el objeto de estudio, presentan como desafío el análisis de sus interrelaciones y conexiones entre diferentes géneros. En efecto, las relaciones y conexiones entre las formas genéricas, es para Swales (2016) uno de los temas actuales más importantes para las lenguas con propósitos específicos. En este marco, es necesario considerar las nociones de macrogénero y microgénero como categorías analíticas relevantes para entender las complejas relaciones entre los géneros. Un macrogénero se define como "una unidad genérica de mayor jerarquía compuesta por géneros" (Venegas, Zamora y Galdames, 2016, p. 252) en cuyo devenir puede incluir variados géneros. Asimismo, un microgénero puede funcionar como un componente de un macrogénero ya sea como incrustados (Rose y Martin, 2012), como partes de un género (Breeze, 2016) o como segmentos retóricos funcionales (Cotos y Chung, 2019). Con base en estos postulados, en los apartados siguientes se presentan y discuten los resultados referidos a la identificación, delimitación y caracterización de los géneros de formación académica desde una perspectiva doble: tipológica y topológica. 


\subsection{Caracterización del macrogénero Informe Técnico en el área de ingeniería civil informática}

La etapa curricular denominada capstone plan o ciclo profesional de carácter terminal se caracteriza por articular un conjunto o set de géneros (Bazerman, 2004) de gran diversidad que los estudiantes de pregrado deben utilizar según su rol en la comunidad de práctica. En este sentido, los hallazgos disponibles en este estudio configuran un mapa o sistema de géneros compuesto por 33 instancias genéricas agrupadas en siete macrogéneros o familias. En la figura 1 se presentan los macrogéneros relevados:
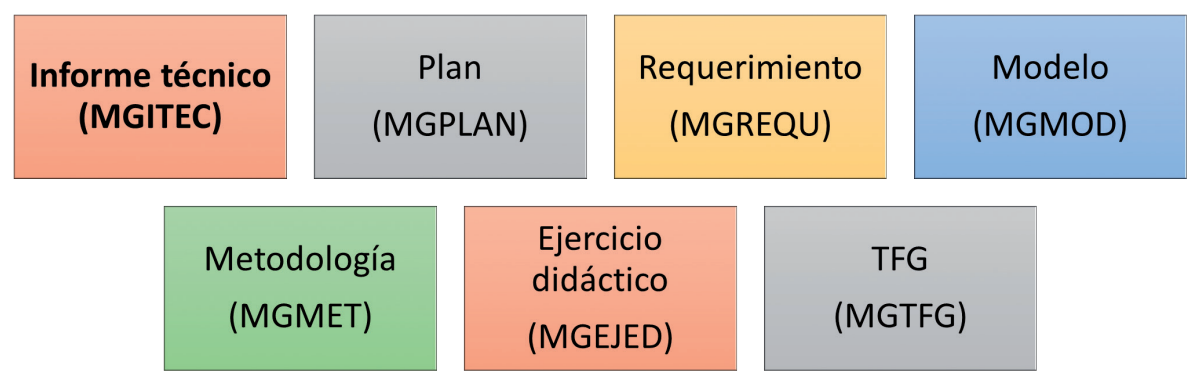

Figura 1. Macrogéneros del discurso académico de la ingeniería civil informática MGEFIC.

Esta amplia diversidad se encuentra refrendada en la literatura disponible sobre la escritura en el ámbito de la ingeniería en otros sistemas de educación superior como el británico (Nesi y Gardner, 2012). En efecto, los tempranos estudios de Nesi y Gardner (2006) y Gardner (2008) han puesto de relieve que la producción escrita en ingeniería es demandante en términos de variación genérica. Por medio de sus investigaciones basadas en corpus y en indagaciones etnográficas en los contextos departamentales (Sologuren, 2019), las investigadoras dan cuenta de que los ingenieros en formación producen tareas de escritura pertenecientes a las 13 familias de géneros identificadas desde ensayos hasta especificaciones de diseño pasando por ejercicios, explicaciones, reportes de investigación, críticas y propuestas, entre otras.

Esto refleja la naturaleza multidisciplinaria de la ingeniería "that draws on disciplines from mathematic to management" (Gardner, 2016, p. 5). También investigaciones sobre el vocabulario de la ingeniería han 
demostrado que existen diferencias significativas entre el vocabulario utilizado entre grupos de diferentes años y ciclos formativos, incluso entre estudiantes de pregrado y postgrado (Durrant, 2016). Este autor plantea que el vocabulario utilizado en cuarto año de ingeniería en contraste con el de tercer año de pregrado es de mayor similitud al utilizado en el ámbito de los negocios. Resultados similares y aún más acentuados obtienen en el ámbito de la salud Castillo y Santos (2020) al comparar el léxico disponible de pacientes diabéticos y con el de profesionales de la salud.

Esto se explica por el aumento sostenido en la focalización que se le brinda a la gestión de los proyectos de ingeniería en los ciclos profesionales y de máster. Asimismo, las investigaciones disponibles en torno al género informe en contexto universitario de pregrado confirman un aumento relevante en la frecuencia de la escritura de informes en los últimos años de ingeniería (Gardner y Holmes, 2009; Parkinson, 2013; Sologuren, 2020a).

El macrogénero Informe técnico en ingeniería civil informática, a partir del análisis del corpus HÉLICE-2017 ha sido definido de la siguiente manera:

Los géneros pertenecientes a esta categoría comparten el macropropósito de consignar el estado de un procedimiento, de un trabajo experimental, de un desarrollo o de un proyecto. Los géneros pertenecientes a esta categoría son los siguientes 15 géneros: IPRO, ISOFT, ILAB, IPP, ICAS, IAL, INV, IEM, IEVAL, IAN, IDIAG, ICON, ITERR e IRREFLEX. Además, esta familia considera el siguiente microgénero: $E F$.

Se trata de un macrogénero que presenta una alta variación genérica y cumple propósitos sociodiscursivos al interior de la comunidad de práctica y formativos:

These types of genres have as their central purpose to give an account of the state of progress of experimental work. It requires students to provide information from one or more sources, analyze such information and provide recommendations about the process developed based on the analysis (Marinkovich, Sologuren y Shawky, 2018, p. 208). 
Escritura académica en tres escuelas de ingeniería chilenas: la familia 'Informe técnico' como macrogénero discursivo en el área de Ingeniería Civil Informática

En efecto, en el interior de este macrogénero convergen géneros de formación que comparten prácticas de escritura que se orientan a familiarizar al estudiante con los conceptos y métodos de la disciplina, es decir, responden más bien a un Texto pedagógico (Gotti, 2014), otras cuyo foco es la escritura de investigación y otras que apuntan hacia un mayor grado de profesionalización que prepare a los estudiantes para los futuros lugares de trabajo.

Los géneros de formación académica en el área de ingeniería civil informática (GEFIC) que pertenecen al macrogénero Informe técnico (MGITEC) son los siguientes:

Tabla 2

Géneros de la familia MGITEC identificados en el corpus de aprendientes con número y código

\begin{tabular}{|l|l|l|l|l|l|}
\hline $\mathbf{N}^{\circ}$ & \multicolumn{1}{c|}{ Código } & \multicolumn{1}{|c|}{ Etiqueta genérica } & \multicolumn{1}{|c|}{$\mathbf{N}^{\circ}$} & \multicolumn{1}{c|}{ Código } & \multicolumn{1}{|c|}{ Etiqueta genérica } \\
\hline 1 & IPRO & Informe de proyecto & 9 & IEV & Informe de evaluación \\
\hline 2 & ISOFT & Informe de software & 10 & IAN & Informe de análisis \\
\hline 3 & ILAB & Informe de laboratorio & 11 & IDIAG & Informe de diagnóstico \\
\hline 4 & IPP & $\begin{array}{l}\text { Informe de práctica } \\
\text { profesional }\end{array}$ & 12 & ICON & Informe de consultoría \\
\hline 5 & ICAS & Informe de caso & 13 & ITERR & Informe de terreno \\
\hline 6 & IAL & Informe de algoritmo & 14 & IRREFLEX & Reporte reflexivo \\
\hline 7 & INV & Informe de investigación & 15 & EF & Estado financiero \\
\hline 8 & IEM & $\begin{array}{l}\text { Informe de estudio de } \\
\text { mercado }\end{array}$ & & & \\
\hline
\end{tabular}

Cada uno de estos 15 géneros han sido identificados, delimitados y definidos a partir de la aplicación de los procedimientos analíticos para el análisis multidimensional de los géneros de formación. En la tabla 3 se presentan los tres géneros más frecuentes en el corpus de aprendientes y que se constituyen por tanto en géneros de formación (Navarro, 2019) prototípicos de esta familia de géneros. El informe de proyecto de carácter fuertemente profesionalizante, el informe de laboratorio altamente académico y el informe de caso que combina una orientación curricular con un carácter profesional. En efecto, el contraste entre los objetivos de cada uno de estos géneros deja en evidencia su hibridación y potencial formativo. 
Escritura académica en tres escuelas de ingeniería chilenas: la familia 'Informe técnico' como macrogénero discursivo en el área de Ingeniería Civil Informática

EnRIQUe SOlOGUREN InsúA

Tabla 3

Objetivos de cada uno de los géneros declarados en su respectivo paso retórico

\begin{tabular}{|c|c|c|}
\hline ILAB & ICAS & IPRO \\
\hline $\begin{array}{l}\text { Paso 3.1: Establecimiento del } \\
\text { propósito }\end{array}$ & $\begin{array}{l}\text { Paso 1.3: Explicitación de } \\
\text { objetivo del informe }\end{array}$ & $\begin{array}{l}\text { Paso 2.2: Presentación de } \\
\text { objetivos del proyecto }\end{array}$ \\
\hline $\begin{array}{l}\text { g. El objetivo de esta Actividad } \\
\text { de Laboratorio es implementar } \\
\text { y analizar algoritmos simples } \\
\text { de detección de movimiento y } \\
\text { tracking de objetos (438-2). }\end{array}$ & $\begin{array}{l}\text { f. Analizar su manera de } \\
\text { funcionar y su modelo de } \\
\text { negocios es primordial para } \\
\text { entender el efecto social y de } \\
\text { mercado que ha generado en } \\
\text { nuestro país la incorporación } \\
\text { de estas aplicaciones }(430-1) \text {. }\end{array}$ & $\begin{array}{l}\text { cc. } 3.1 \text { Objetivo General } \\
\text { Realizar un seguimiento } \\
\text { continuo del estado de salud } \\
\text { del usuario a través de una } \\
\text { aplicación y una pulsera } \\
(166-3) \text {. }\end{array}$ \\
\hline
\end{tabular}

Mientras que ILAB, que se encuentra más en el polo de la escritura de investigación, se corresponde de forma más prototípica con una actividad curricular en el aula de ingeniería, ICAS por su parte avanza en el desarrollo de una competencia reflexiva y, por consiguiente, presentan rasgos mixtos que lo acercan a una escritura profesional, pero también incorpora el componente de construcción analítica y crítica que promueve el curriculum, para la formación en ingeniería y ciencias. De este modo, formar profesionales capaces de "modelar, diseñar, evaluar, implementar y gestionar sistemas informáticos, apoyado en las Tecnologías de la Información y las Comunicaciones (USM, 2020).

Tabla 4

Géneros más frecuentes de la familia MGITEC con número y código

\begin{tabular}{|c|c|c|l|}
\hline$N^{\circ}$ & Género & Código & \multicolumn{1}{c|}{ Definición } \\
\hline 1 & $\begin{array}{c}\text { Informe de } \\
\text { proyecto }\end{array}$ & IPRO & $\begin{array}{l}\text { Género discursivo de formación cuyo propósito comunicativo es dar cuenta } \\
\text { de un proyecto o desarrollo de una idea y persuadir sobre su relevancia. Su } \\
\text { organización discursiva predominante es argumentativa y descriptiva. Se } \\
\text { configura semióticamente de forma preferente a través del modo verbal y } \\
\text { gráfico. La relación entre los participantes es escritor semilego - lector } \\
\text { experto o lector semilego. El contexto de circulación es laboral. Su objetivo } \\
\text { de aprendizaje es aplicar conocimientos en nuevos contextos. }\end{array}$ \\
\hline 5 & $\begin{array}{l}\text { Informe de } \\
\text { caso }\end{array}$ & ICAS & $\begin{array}{l}\text { Género discursivo de formación cuyo propósito comunicativo es exponer un } \\
\text { estudio de caso o estudios de casos de implementación y experimentación. } \\
\text { Su organización discursiva predominante es explicativa. Se configura } \\
\text { semióticamente de forma preferida a partir del modo verbal y gráfico. La } \\
\text { relación entre los participantes es escritor semilego - lector experto. El } \\
\text { contexto de circulación es pedagógico. Su objetivo de aprendizaje es } \\
\text { analizar y aplicar conceptos teóricos a situaciones concretas. }\end{array}$ \\
\hline 3 & $\begin{array}{l}\text { Informe de } \\
\text { laboratorio }\end{array}$ & ILAB & $\begin{array}{l}\text { Género discursivo de formación cuyo propósito comunicativo es constatar } \\
\text { fenómenos observados e interpretarlos en condiciones controladas. Su } \\
\text { organización discursiva predominante es descriptiva. Se configura } \\
\text { semióticamente de forma preferente a través del modo verbal, gráfico y } \\
\text { matemático. La relación entre los participantes es escritor semilego -lector } \\
\text { experto. El contexto de circulación es científico. Su objetivo de aprendizaje } \\
\text { implicado es el analizar y aplicar procedimientos propios del método } \\
\text { científico. }\end{array}$ \\
\hline
\end{tabular}


Escritura académica en tres escuelas de ingeniería chilenas: la familia 'Informe técnico' como macrogénero discursivo en el área de Ingeniería Civil Informática

Finalmente, el informe de proyecto dada su alta estabilidad y tal como se puede apreciar en el ejemplo de objetivo de la tabla 3 se trata del género de formación GEFIC más apegado al mundo profesional futuro que el ingeniero en formación encontrará una vez concluido el ciclo terminal. En este último ámbito de orientación claramente profesional encontramos las siguientes clases de informes:

Tabla 5

Géneros +NO académicos de la familia MGITEC con número y código

\begin{tabular}{|c|c|c|c|}
\hline $\mathbf{N}^{\circ}$ & Género & Código & Defĩnición \\
\hline 2 & $\begin{array}{l}\text { Informe de } \\
\text { software }\end{array}$ & ISOFT & $\begin{array}{l}\text { Género discursivo de formación cuyo propósito comunicativo es presentar un determinado } \\
\text { sofiware o aplicación desarrollada. Su organización discursiva predominante es explicativa. Se } \\
\text { configura semióticamente de forma preferente a través del modo verbal y gráfico. La relación } \\
\text { entre los participantes es escritor semilego - escritor experto y semilego. El contexto de } \\
\text { circulación es laboral. Su objetivo de aprendizaje es aplicar conceptos, principios y teorías, en el } \\
\text { marco del desarrollo de determinadas técnicas y procedimientos. }\end{array}$ \\
\hline 8 & $\begin{array}{l}\text { Informe de } \\
\text { estudio de } \\
\text { mercado }\end{array}$ & IEM & $\begin{array}{l}\text { Género discursivo de formación cuyo propósito comunicativo es caracterizar el mercado en un } \\
\text { determinado sector y en un período determinado. Su organización discursiva predominante es } \\
\text { descriptiva. Se configura semióticamente de forma preferida a través del modo verbal y gráfico. } \\
\text { La relación entre los participantes es escritor semilego - lector experto. El contexto de circulación } \\
\text { es laboral. Su objetivo de aprendizaje es analizar un objeto o proceso económico y aplicar } \\
\text { conocimientos que permitan relevar la significancia del estudio. }\end{array}$ \\
\hline 15 & $\begin{array}{l}\text { Estado } \\
\text { financicro }\end{array}$ & $\mathrm{EF}$ & $\begin{array}{l}\text { Microgénero discursivo de formación cuyo propósito comunicativo es dar cuenta de la situación } \\
\text { económica y financicra de una institución o empresa en un periodo determinado de tiempo. Su } \\
\text { organización discursiva predominante es descriptiva. Se configura semióticamente de forma } \\
\text { preferida a través del modo gráfico y del modo matemático. La relación entre los participantes es } \\
\text { escritor semilego y experto - lector experto. El contexto de circulación es laboral. Su objetivo de } \\
\text { aprendizaje es analizar y comprender las transacciones del negocio. }\end{array}$ \\
\hline 9 & $\begin{array}{l}\text { Informe de } \\
\text { evaluación }\end{array}$ & IEVAL & $\begin{array}{l}\text { Género discursivo de formación cuyo propósito comunicativo es reportar la evaluación } \\
\text { desarrollada de un sitio web, un producto, un software, un videojuego, un sistema, entre otros. Su } \\
\text { organización discursiva predominante es argumentativa. Se configura semióticamente de forma } \\
\text { preferida a través del modo verbal y gráfico. La relación entre los participantes es escritor } \\
\text { semilego - lector experto y semilego. El contexto de circulación es laboral. Su objetivo de } \\
\text { aprendizaje es comprender globalmente el funcionamiento y comportamiento de un determinado } \\
\text { elemento para analizar sus características y evaluar su diseño, composición, usabilidad, entre } \\
\text { otros aspectos relevantes. }\end{array}$ \\
\hline 10 & $\begin{array}{l}\text { Informe de } \\
\text { análisis de } \\
\text { negocio }\end{array}$ & $\mathrm{IAN}$ & $\begin{array}{l}\text { Género discursivo de formación cuyo propósito comunicativo es reportar el análisis preliminar } \\
\text { que permitirá diseñar un modelo de negocios. Su organización discursiva predominante es } \\
\text { descriptiva. Se configura semióticamente a través del modo verbal y gráfico de forma preferente. } \\
\text { La relación entre los participantes es escritor semilego - lector experto. El contexto de circulación } \\
\text { es laboral. Su objetivo de aprendizaje es analizar y evaluar un escenario económico con miras a } \\
\text { la implementación de un determinado modelo de negocio. }\end{array}$ \\
\hline 11 & $\begin{array}{l}\text { Informe de } \\
\text { diagnóstico }\end{array}$ & IDIAG & $\begin{array}{l}\text { Género discursivo de formación cuyo propósito comunicativo es explicar los resultados de un } \\
\text { proceso de diagnóstico en torno a un determinado problema en el ámbito de la informática y la } \\
\text { sociedad. Su organización discursiva predominante es argumentativa. Se configura } \\
\text { semióticamente de forma preferente a través del modo verbal y gráfico. La relación entre los } \\
\text { participantes es escritor semilego - lector experto y semilego. El contexto de circulación es } \\
\text { laboral. Su objetivo de aprendizaje consiste en evaluar de forma global un problema mediante el } \\
\text { análisis y la argumentación. }\end{array}$ \\
\hline 12 & $\begin{array}{l}\text { Informe de } \\
\text { consultoría }\end{array}$ & ICON & $\begin{array}{l}\text { Género discursivo de formación cuyo propósito comunicativo es consignar los resultados y } \\
\text { observaciones derivados de un proceso de consultoría. Su organización discursiva predominante } \\
\text { es explicativa y argumentativa. Se configura semióticamente de forma preferente a través del } \\
\text { modo verbal y gráfico. La relación entre los participantes es escritor semilego y experto - lector } \\
\text { experto. El contexto de circulación es laboral. Su objetivo de aprendizaje es analizar, sintetizar y } \\
\text { evaluar información con el objeto de desarrollar una recomendación experta. }\end{array}$ \\
\hline
\end{tabular}


Escritura académica en tres escuelas de ingeniería chilenas: la familia 'Informe técnico' como macrogénero discursivo en el área de Ingeniería Civil Informática

En contraste, como era de esperar en este ciclo, los tipos de informes asociados a la escritura de investigación conforman un grupo más reducido, los que son definidos en la siguiente tabla 6:

Tabla 6

Géneros +Académicos de la familia MGITEC con número y código

\begin{tabular}{|c|c|c|c|}
\hline $\begin{array}{c}\mathbf{N} \\
\text { o }\end{array}$ & Género & Código & \multicolumn{1}{c|}{ Definición } \\
\hline 6 & $\begin{array}{c}\text { Informe de } \\
\text { Algoritmo }\end{array}$ & IAL & $\begin{array}{l}\text { Género discursivo de formación cuyo propósito comunicativo es consignar } \\
\text { el desarrollo de un algoritmo. Su organización discursiva predominante es } \\
\text { explicativa. Se configura semióticamente de forma preferida a través del } \\
\text { modo verbal y matemático. La relación entre los participantes es escritor } \\
\text { semilego - lector experto. El contexto de circulación es científico. Su } \\
\text { objetivo de aprendizaje es analizar y procesar información, con base en la } \\
\text { interpretación y razonamiento hipotético-deductivo. }\end{array}$ \\
\hline 7 & $\begin{array}{c}\text { Informe de } \\
\text { investigación }\end{array}$ & INV & $\begin{array}{l}\text { Género discursivo de formación cuyo propósito comunicativo es reportar } \\
\text { resultados y aprendizajes de un determinado proceso investigativo } \\
\text { desarrollado. Su organización discursiva predominante es explicativa. Se } \\
\text { configura semióticamente de forma preferida a través del modo verbal y } \\
\text { gráfico. La relación entre los participantes es escritor semilego - lector } \\
\text { experto y semilego. El contexto de circulación es científico. Su objetivo de } \\
\text { aprendizaje implicado es comprender el alcance y relevancia de un tópico } \\
\text { en específico para el área de estudio concemida. }\end{array}$ \\
\hline
\end{tabular}

Finalmente, esta familia de géneros se completa con dos géneros naturaleza híbrida en tanto que al igual que el informe de caso (ICAS) presentan una naturaleza dual: curricular y profesional, en el que lo práctico y lo epistémico se encuentra disociado (Anson, 2002):

Tabla 7

Géneros + Híbridos de la familia MGITEC con número y código

\begin{tabular}{|c|c|c|c|}
\hline $\begin{array}{c}\mathbf{N} \\
0\end{array}$ & Género & Código & \multicolumn{1}{c|}{ Definición } \\
\hline 13 & $\begin{array}{c}\text { Informe de } \\
\text { terreno }\end{array}$ & ITERR & $\begin{array}{l}\text { Género discursivo de formación cuyo propósito comunicativo es dar cuenta } \\
\text { de las observaciones desarrolladas durante el trabajo de campo. Su } \\
\text { organización discursiva predominante es descriptiva. Se configura } \\
\text { semióticamente de forma preferida a través del modo verbal y gráfico. La } \\
\text { relación entre los participantes es escritor semilego - lector experto. El } \\
\text { contexto de circulación es científico. Su objetivo de aprendizaje subyacente } \\
\text { es comprender globalmente un problema integrando la observación y el } \\
\text { análisis. }\end{array}$ \\
\hline 4 & $\begin{array}{c}\text { Informe de } \\
\text { práctica } \\
\text { profesional }\end{array}$ & IPP & $\begin{array}{l}\text { Género discursivo de formación cuyo propósito comunicativo es exponer } \\
\text { los principales resultados y aprendizajes del proceso de práctica } \\
\text { preprofesional. Su organización discursiva predominante es explicativa. Se } \\
\text { configura semióticamente de forma preferente a partir del modo verbal y } \\
\text { gráfico. La relación entre los participantes es escritor semilego - lector } \\
\text { experto y semilego. El contexto de circulación es pedagógico. Su objetivo } \\
\text { de aprendizaje subyacente es aplicar conocimientos procedimentales } \\
\text { específicos en la resolución de problemas profesionales, así como promover } \\
\text { la reflexión en torno a los procesos implicados. }\end{array}$ \\
\hline
\end{tabular}


Escritura académica en tres escuelas de ingeniería chilenas: la familia 'Informe técnico' como macrogénero discursivo en el área de Ingeniería Civil Informática

ENRIQUE SOlOgUREN INSÚA

A partir de los datos obtenidos es posible identificar un continuum de géneros discursivos que van desde informes orientados a la inserción académica en la ingeniería hasta la producción escrita de informes orientados a contextos profesionales. En efecto, en esta familia se despliega una amplia variedad de tipos de informes que cumplen propósitos formativos de relevancia en la aplicación de pensamiento analítico, crítico y creativo para la resolución de problemas: la raison d'être de la ingeniería.

En este continuum representado en la figura 2 se observa la emergencia de redes de géneros de formación o epistémicos de naturaleza híbrida. Una red de géneros en los términos propuestos por Swales (2004) busca captar alguna noción de cuadro general vigente o panorama global de una forma más dinámica que la que provee el constructo bazermaniano de sistema de géneros. Las redes de géneros refieren a relaciones intertextuales más amplias entre géneros en un determinado campo de actividad y en una determinada comunidad de aprendizaje. En definitiva, capturan las relaciones entre los géneros que son siempre dinámicas y en constante evolución.

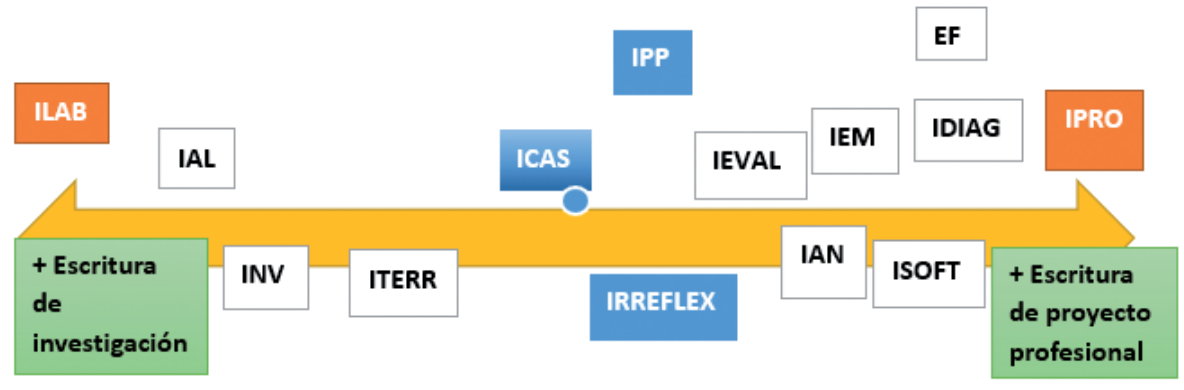

Figura 2. Continuum de los géneros que conforman el macrogénero Informe Técnico (MGITEC) de Ingeniería Civil Informática.

Así las cosas, en la figura 2 podemos apreciar una red de géneros de fuerte orientación académica o investigativa compuesta por cuatro géneros que se aproximan hacia un polo netamente académico: ILAB, IAL, INV e ITERR, pero que presentan rasgos híbridos propios de un espacio textual (Bhatia, 2010) altamente dinámico debido a la confluencia de diferentes disciplinas, prácticas de escritura específicas y culturas académicas y profesionales: "Expert professional writers constantly operating within and across generic boundaries creating new but essentially rela- 
ted and/or hybrid (both mixed and embedded) forms to give expression to their 'private intentions' within the socially accepted communicative practices and shared generic norms" (Bhatia, 2012, p. 24).

En el otro extremo del continuum se configura una red numerosa de géneros de orientación profesional no académica actualizados en versiones pedagógicamente orientadas para el desarrollo de diversos tipos de habilidades esperadas en el ámbito laboral. Estos siete géneros (tabla $N^{\circ} 5$ ), responden no solo a la línea curricular de ingeniería aplicada, sino que también a la educación en gestión, finanzas, economía y administración de proyectos. En efecto, los objetivos educacionales de estos cursos son concordantes con el siguiente atributo del graduado en ingeniería de base científica que reconoce el acuerdo de Washington:

WA11: Demonstrate knowledge and understanding of engineering management principles and economic decision-making and apply these to one's own work as a member and leader in a team, to manage projects and in multi-disciplinary environments (IEA, 2014: 15).

En cada uno de estos espacios textuales o constelaciones (Swales, 1990) que circulan en un momento determinado y en un ciclo formativo específico (terminal), los diferentes miembros del macrogénero informe técnico establecen diferentes tipos de relaciones de incrustación, mezcla y conexión, cuya comprensión y puesta en práctica posibilita la apropiación de los recursos genéricos: "Appropriation of generic resources is also very common in various form of hybrids, such as mixing, embedding and bending of genres" (Bhatia: 2012, p. 25). Así, un ingeniero en formación como participante periférico legítimo (Lave y Wenger, 2001) de una comunidad de práctica tendrá que tomar conciencia de que los géneros no funcionan de forma aislada y que su adquisición durante el trayecto de aprendizaje es vital para lograr desplazarse hacia posiciones de mayor compromiso de tiempo y esfuerzo al interior de las comunidades de práctica y, en consecuencia, de construcción de la identidad profesional (Noceti y Benedetti, 2010).

Sumado a esto, en el centro del continuum del MGITEC se ubican tres géneros de fuerte naturaleza curricular, esto es, que se orientan hacia: "goal driven classroom activities, devoted to the accomplishment of significant educational ends" (Christie, 2002, p. 22). En este sentido, se trata de géneros en los que sus posibles versiones expertas en las comunidades académicas y profesionales son más difusas. En este sentido, se trata de recursos genéricos propios de los contextos instruccionales y adscri- 
Escritura académica en tres escuelas de ingeniería chilenas: la familia 'Informe técnico' como macrogénero discursivo en el área de Ingeniería Civil Informática

ENRIQUE SOlOgUREN INSÚA

tos al discurso del aula (Cotos y Chung, 2019) en un sentido amplio.

De este modo, el informe de caso, el informe de práctica profesional y el reporte reflexivo constituyen una preparación útil para la escritura en los lugares de trabajo, pero su foco está puesto en la aplicación de conceptos clave de la disciplina, en los métodos de trabajo y en la reflexión generada a partir del análisis a casos reales o situaciones profesionales. ICAS, por su parte, puede presentar aún mayor hibridez, ya que, si es parte de un análisis de negocio, puede integrarse al género informe de análisis de negocio que perfila una audiencia de corte profesional y busca persuadir al lector para que tome una decisión en el ámbito financiero (Yeung, 2007). En este sentido, cobran especial relevancia trabajos como el de Romero, Trigo y Álvarez (2020) que fijan su foco de atención en el dominio de los recursos visuales al presentar el informe ante un público -profesional o académico-, pues será clave para lograr una efectiva persuasión de la audiencia.

\section{Conclusiones y proyecciones}

Este artículo se planteó como objetivo describir la familia genérica 'Informe Técnico' en la escritura estudiantil en tres escuelas chilenas de ingeniería civil informática. Con base en estos resultados, se perfilan tres subfamilias genéricas 1) +investigación, 2) +curricular y 3) + profesional cada una representada por el género de mayor prototipicidad, relevancia y grado de presencia en el corpus de aprendientes. Estos géneros, que actúan como núcleos en cada una de sus redes, los hemos categorizado al interior del macrogénero informe técnico como de mesonivel en tanto comparten características retóricas y discursivas con los integrantes de sus respectivas constelaciones, así como propósitos comunicativos similares y audiencias específicas. Estos mesogéneros posibilitan el desarrollo de una investigación en términos de su diseño, ejecución y apreciación (ILAB), la comprensión de las prácticas profesionales (ICAS), así como la organización de un curso de acción (IPRO).

Los mesogéneros que se ubican en los extremos del continuum ILAB e IPRO se encuentran presentes en los subcorpus de cada de las tres universidades del estudio. ICAS, por su parte, está presente en dos de tres subcorpus. Esto da cuenta de que se trata de prácticas discursivas extendidas en la cultura disciplinar y que no pertenecen exclusivamente 
a una unidad académica en específico. Además, esto nos indica que la escritura reflexiva (Breuer, Newman y Newman, 2016; Klein y Boscolo, 2016) no ha permeado del todo los procesos formativos en esta cultura disciplinar. Esto exige, además, como proyección del estudio, el levantamiento de cada uno de los mesomodelos retórico-discursivos para así lograr una mirada interdisciplinaria sobre los géneros de formación (Castillo y Sologuren, 2020; Sologuren, 2020b).

También este macrogénero pretende aportar en las habilidades de modelamiento y aplicación de conocimientos como lo exigen, por ejemplo, los estándares internacionales en la formación de ingenieros: "an ability to apply knowledge of mathematics, science, and engineering" (ABET, 2017, p. 3). Así como de transferencia de los aprendizajes (Parodi, 2014).

Todos los elementos hasta acá analizados tienen consecuencias relevantes para la alfabetización profesional que deben ser exploradas y profundizadas en conjunto con la descripción de cada uno de los GEFICs que forman nuestro sistema. Los géneros profesionales estudiantiles, permiten, desde esta perspectiva, aprender para comunicar y comunicar para aprender en los términos propuestos por Carlsson, Malmström y Edström (2010) por medio de la aplicación de pensamiento crítico y creativo (Jarpa, 2019). A esto se suma, la indagación de patrones retóricos preferidos (Connor, 2013) en los textos estudiantiles de esta subdisciplina de la ingeniería para así entender de mejor forma de qué manera se constituye una escritura académica exitosa en esta disciplina y a partir de ello andamiar procesos de alfabetización disciplinar en el curriculum de ingeniería civil.

\section{Referencias}

ABET. (2017). Criteria for accrediting engineering programs. Recuperado de https://www. abet.org/wp-content/uploads/2015/10/E001-16-17-EAC-Criteria-10-20-15.pdf

Amieva, R. L. (2001). Elaboración de informes en la enseñanza de la ingeniería. Facultad de Ingeniería. Gabinete de Asesoramiento Pedagógico. Universidad Nacional de Río Cuarto. Recuperado de http://www.ehu.eus/pat/compe/lanak/elaboracion_de_informes_en_la_ensennanza_de_la_ingenieria_informes_lengua $\% 20 \mathrm{y} \% 20$ contenido.pdf

Anson, C. M. (2002). The WAC casebook: Scenes for faculty reflection and program development.

Angouri, J. (2010). Quantitative, qualitative or both? Combining methods in linguistic research. In L. Litosseliti (Ed.), Research methods in linguistics (pp. 29-45). New York: Continuum. 
Escritura académica en tres escuelas de ingeniería chilenas: la familia 'Informe técnico' como macrogénero discursivo en el área de Ingeniería Civil Informática

EnRIQUe SOLOGURen Insúa

Arechabala, M., Catoni, M., Ávila, N., Riquelme, G. \& Aedo, V. (2011). Discourse genres and most frequent mistakes in academic reports of nursing students. Investigación y Educación en Enfermería, 29(3), 400-406.

Artemeva, N. (2009). Stories of becoming: A study of novice engineers learning genres of their profession. In C. Bazerman, A. Bonini, \& D. Figueiredo (Eds.), Genre in a changing world (pp. 158-178). Fort Collins, CO: WAC Clearing house and Parlor Press.

Ávila, N. \& Cortés, A. (2017). El género" informe de caso" en la formación inicial docente: una aproximación basada en la actividad. Lenguas Modernas, (50), 153-174.

Bazerman, C. (2004). Intertextuality: How texts rely on other texts. In C Bazerman \& P. Prior (Eds.), What writing does and how it does it: An introduction to analyzing texts and textual practices, (pp. 83-96). Mahwah, NJ: Lawrence Erlbaum Associates.

Bhatia, V. (2010). Interdiscursivity in professional discourse. Discourse \& Communication, $4(1), 32-50$.

Bhatia, V. (2012). Critical reflections on genre analysis. Ibérica, (24), 17-28.

Bhatia, V. (2016). Critical genre analysis: Investigating interdiscursive performance in professional practice. London: Routledge.

Breeze, R. (2016). Tracing the development of an emergent part-genre: The author summary. English for Specific Purposes, 42, 50-65.

Breuer, E., Newman, S. \& Newman, J. (2016). Learning to write as a professional: Engineers and health professionals in the United Kingdom and Germany. In G. Ortoleva, M. Bétrancourt \& S. Billett (Eds.), Writing for professional development (pp. 253-275). Leiden, The Netherlands: Brill.

Carlsson, C., Malmström, H. \& Edström, K. (2010). Engineering and Communication Integrated Learning - Collaboration Strategies for Skills and Subject Experts. Proceedings of the 6th international CDIO conference, École Polytechnique, Montréal, Canada.

Cassany, D. \& López, C. (2010). De la universidad al mundo laboral: continuidad y contraste entre las prácticas letradas académicas y profesionales. En G. Parodi (Ed.), Alfabetización académica y profesional en el siglo XXI. Leer y escribir desde las disciplinas (pp. 347-374). Santiago: Ariel.

Castillo Fadic, M. N. (2020). Corpus Básico del Español de Chile C: metodología de obtención, revisión y constitución definitiva. BFUCh: en prensa.

Castillo Fadic, M. N. y E. Sologuren (2020). Léxico frecuente, riqueza léxica y estereotipos sobre la lectura de profesores en formación. Logos: Revista de Lingüística, Filosofía y Literatura, 30(1): 69-85.

Castillo Fadić, María Natalia. Inmaculada Santos Díaz. (2020). “Dos miradas sobre el cuerpo humano en el marco de la literacidad en salud: profesionales de la salud y personas con diabetes mellitus 2". En Maribel Serrano Zapata y $M^{a}$ Ángeles Carrero Fernández (ed.), Aplicaciones de la disponibilidad léxica, Universitat de Lleida, Lleida.

Christie, F. (2002). Classroom Discourse Analysis: a functional perspective. London: Continuum.

Connor, U. (2013). ESP and intercultural rhetoric. In B. Paltridge \& S Starfield (Eds.), The handbook of English for specific purposes (pp. 427-443). West Essex, UK: WileyBlackwell.

Corbetta, P. (2007). Metodología y técnicas de investigación social. Madrid: McGraw-Hill. 
Escritura académica en tres escuelas de ingeniería chilenas: la familia 'Informe técnico' como macrogénero discursivo en el área de Ingeniería Civil Informática

ENRIQUe SOLOGUREN INSÚA

Carpio, G. C., \& Carlino, P. (2019). Qué hace un docente de Ingeniería para promover la participación de sus alumnos en la construcción de conocimientos: escritura, revisión colectiva y diálogo sobre lo escrito. Cuaderno de Pedagogía Universitaria, 16(32), 4-18.

Cotos, E. \& Chung, Y. (2019). Functional language in curriculum genres: Implications for testing international teaching assistants. Journal of English for Academic Purposes, 41, 100766.

Da Cunha, I. \& Montané, M. A. (2019). Géneros textuales y dificultades de redacción en ámbitos especializados. Revista Signos, 52(99), 4-30.

Durrant, P. (2016). To what extent is the Academic Vocabulary List relevant to university student writing? English for Specific Purposes, 43, 49-61.

Ferreira, A., Elejalde, J. \& Vine, A. (2014). Análisis de errores asistido por computador basado en un corpus de aprendientes de español como lengua extranjera. Revista Signos, 47(86), 385-411.

Gardner, S. (2008). Integrating Ethnographic, Multidimensional, Corpus Linguistic and Systemic Functional Approaches to Genre Description: An Illustration through University History and Engineering Assignments. In E. Steiner \& S. Neumann (Eds.), Data and Interpretation in Linguistic Analysis. 19th European SFL Conference July 2007 (pp. 1-34) Saarbrücken: Universität des Saarlandes.

Gardner, S. (2012). Genres and registers of student report writing: An SFL perspective on texts and practices. Journal of English for Academic Purposes, 11(1), 52-63.

Gardner, S. (2016). A genre-instantiation approach to teaching English for specific academic purposes: Student writing in business, economics and engineering. Writing and Pedagogy, 8(1), 117-144.

Gardner, S. \& Holmes, J. (2009), Can I use headings in my essay? Section headings, macrostructures and genre families in the BAWE corpus of student writing. In M. Charles, S. Hunston. \& D. Pecorari (Eds.), Academic writing: At the interface of corpus and discourse (pp. 251-271). London: Continuum.

Gardner, S. \& Nesi, H. (2013). A classification of genre families in university student writing. Applied Linguistics, 34, 25-52.

Gilmore, A. \& Millar, N. (2018). The language of civil engineering research articles: A corpus-based approach. English for Specific Purposes, 51, 1-17.

González C. \& Burdiles, G. (2018). Rhetorical organization of the genre Financial Stability Report: a contrast between the report of the Central Bank of Chile and the German Federal Bank. Círculo de Linguistica Aplicada a la Comunicación, (73), 145-160.

Gotti, M. (2014). Reformulation and recontextualization in popularization discourse. Ibérica, Revista de la Asociación Europea de Lenguas para Fines Específicos, (27), 15-34.

Harvey, A. (Comp.) (2005). En torno al discurso: Contribuciones de América Latina. Santiago de Chile: Ediciones Universidad Católica de Chile.

Harvey, A. \& Muñoz, D. (2006). El género informe y sus representaciones en el discurso de los académicos. Estudios filológicos, 41, 95-114.

Ibáñez, R., Moncada, F., Cornejo, F., \& Arriaza, V. (2017). Los Géneros del Conocimiento en Textos Escolares de educación primaria. Calidoscópio, 15(3), 462-476.

International Engineering Alliance. (2014). Graduate attributes and professional competencies. Retrieved from https://www.ieagreements.org/accords/washington/ 
Escritura académica en tres escuelas de ingeniería chilenas: la familia 'Informe técnico' como macrogénero discursivo en el área de Ingeniería Civil Informática

EnRIQUe SOLOGURen Insúa

Jarpa, M. (2019). Escritura académica para el desarrollo de la reflexión pedagógica en la formación docente: la crónica del docente-directivo. Íkala. Revista de lenguaje y cultura, 24(1), 85-101.

Klein, P. \& Boscolo, P. (2016). Trends in research on writing as a learning activity. Journal of Writing Research, 7(3), 311-350.

Lave, J. \& Wenger, E. (2001). Situated Learning: legitimate peripheral participation (reimpresión). New York: Cambridge University Press.

Lee, D. \& Swales, J. (2006). A corpus-based EAP course for NNS doctoral students: Moving from available specialized corpora to self-compiled corpora. English for specific purposes, 25(1), 56-75.

Marinkovich, J., Sologuren, E. \& Shawky, M. (2018). The process of academic literacy in Civil Engineering Computer Science. An approach to academic writing and its genres in a learning community. Círculo de Lingüística Aplicada a la Comunicación, 74, 195-220.

Martin, J. \& Rose, D. (2007). Working with Discourse. Meaning Beyond the Clause. $2^{\text {nd }}$ edition. London: Continnuum.

Molina, M., Vásquez-Rocca, L. \& Parodi, G. (2018). Relación palabra-gráfico en un género profesional de la economía: el Informe Mensual de Estadísticas Monetarias y Financieras. Círculo de Lingüística Aplicada a la Comunicación, 76, 153-178.

Montolío, E. (2010). Mejorar las recomendaciones contenidas en los informes elaborados por consultores. La optimización del discurso. Onomazéin, 21(1), 237-253.

Montolío, E. \& López, A. (2010). Especificidades discursivas de los textos profesionales frente a los textos académicos: El caso de la recomendación profesional. En G. Parodi (Ed.), Alfabetización académica y profesional en el siglo XXI: Leer y escribir desde las disciplinas (pp. 215-245). Santiago: Ariel.

Morales, O. A., Perdomo, B, Cassany, D. \& Izarra, É. (2020). Estructura retórica de tesis y trabajos de grado en odontología: un estudio en hispanoamérica y España. Íkala. Revista de lenguaje y cultura, 25(2), 373-393.

Moreno de León, T. (2017). Análisis retórico funcional del género profesional Informe Anual del Fondo Monetario Internacional. Trabajo presentado en el Tercer Simposio Internacional de la Cátedra UNESCO Lectura y Escritura: tensiones y desafíos en su enseñanza e investigación. Chillán, Chile.

Muñoz, D. (2006). Estructura y patrones léxicos en informes escritos de estudiantes universitarios. Onomázein, 13(1), 55-71.

Nathan, P. (2016). Analyzing options in pedagogical business case reports: Genre, process and language. English for Specific Purposes, 44, 1-15.

Navarro, F. (2019). Aportes para una didáctica de la escritura académica basada en géneros discursivos. DELTA: Documentação de Estudos em Lingüística Teórica e Aplicada, 35(2).

Nesi, H. \& Gardner, S. (2006). Variation in disciplinary culture: university tutors'views on assessed writing tasks. In R. Kiely, P. Rea-Dickins, H. Woodfield, \& G. Clibbon (Eds.), Language, culture and identity in applied linguistics (pp. 99-117). London: BAAL/ Equinox.

Nesi, H. \& Gardner, S. (2012). Genres across the disciplines. Student writing in higher education. Cambridge: Cambridge University Press. 
Escritura académica en tres escuelas de ingeniería chilenas: la familia 'Informe técnico' como macrogénero discursivo en el área de Ingeniería Civil Informática

ENRIQUe Sologuren InsúA

Noceti, A. \& Benedetti, M. (2010). Socialización, entrenamiento y educación en ingeniería mediante sistemas de géneros: una experiencia integradora.

Oyarzún, K. (2016). Aproximación a la descripción del discurso profesional de la economía a través del Informe Económico Regional (Tesis de Licenciatura en Lengua y Literatura Hispanoamericana). Pontificia Universidad Católica de Valparaíso.

Pagano R. (2012) Understanding statistics in the behavioral sciences. Belmont, CA: Cengage.

Parkinson, J. (2013). Adopting academic values: Student use of that-complement clauses in academic writing. System, 41(2), 428-442.

Parkinson, J. (2017). The student laboratory report genre: A genre analysis. English for Specific Purposes, 45, 1-13.

Parodi, G. (2014). Comprensión de textos escritos. Teoría de la Comunicabilidad. Buenos Aires: Eudeba.

Parodi, G., Ibáñez, R., Venegas, R. \& González, C. (2010). Identificación de géneros académicos y géneros profesionales: Principios teóricos y propuesta metodológica. En G. Parodi (Ed.), Alfabetización académica y profesional en el siglo XXI: Leer y escribir desde las disciplinas (pp. 249-289). Santiago de Chile: Ariel.

Parodi, G., Julio, C. \& Vásquez-Rocca, L. (2015). Los géneros del Corpus PUCVUCSC-2013 del discurso académico de la economía: el caso del Informe de Política Monetaria. Revista ALED, 15(3), 179-200.

Quacquarelli Symonds (QS) (2019). World University Rankings. Recuperado de http:// www.qs.com/ranking.html

Rea Rizzo, C. (2010). A first approach to the lexical profile of telecommunication English: frequency, distribution, restriction and keyness. Revista de Lingüística y Lenguas Aplicadas, 4(1), 161-173.

Rodgers, O., Chambers, A. \& Le Baron-Earle, F. (2011). Corpora in the LSP classroom: A learner-centred corpus of French for biotechnologists. International Journal of Corpus Linguistics, 16(3), 391-411.

Romero, M. F., Salvador, A. \& Trigo, E. (2014). Propuestas para trabajar la escritura académica en los estudios universitarios. En M. F. Romero (Coord.) La escritura académica: diagnóstico y propuestas de actuación. Una visión desde los grados de magisterio (pp. 87-100). Barcelona: Octaedro.

Romero, M. F., Trigo, E. \& Álvarez, E. (2020). Apoyos visuales para la defensa del trabajo: la presentación digital. En M. F. Romero (Ed.) Escribir en la universidad. Elaboración y defensa de trabajos académicos -TFG/TFM- (pp. 123-142). Berlín: Peter Lang.

Rose, D. \& Martin, J. (2012). Learning to write, reading to learn. Genre, knowledge and pedagogy in the Sydney School. London: Equinox.

Sologuren, E. (2019). Approche de l'écrit académique dans un milieu d'apprentissage de I'Ingénierie Civile en Informatique. Policromias-Revista de Estudos do Discurso, Imagem e Som, 4(2), 52-65.

Sologuren, E., \& Castillo, M. (2019). La construcción del Ethos en informes de laboratorio producidos por estudiantes universitarios: contrastes en el discurso académico en español. Letras de Hoje, 54(3), 369-384.

Sologuren, E. (2020). Maps of student genres in engineering: a didactic model for tea- 
Escritura académica en tres escuelas de ingeniería chilenas: la familia 'Informe técnico' como macrogénero discursivo en el área de Ingeniería Civil Informática

ENRIQUE SOlOGUREN INSÚA

ching academic and professional Spanish language. Language Value 12. https://doi. org/10.6035/LanguageV.2020.12.6

Sologuren Insúa, E. (2020). Géneros de formación en el ciclo capstone de Ingeniería Civil Informática: Exploraciones al curriculum. Revista de estudios y experiencias en educación, 19(41), 167-198.

Swales, J. (1990). Genre analysis: English in academic and research settings. Cambridge University Press.

Swales, J. (1996). Occluded genres in the academy: the case of the submission letter. In E. Ventola \& A. Mauranen (Eds.), Academic Writing: Intercultural and Textual Issues. Amsterdam: John Benjamins.

Swales, J. (2004). Research genres: Explorations and applications. Cambridge: Cambridge University Press.

Swales, J. (2016). Configuring image and context: Writing 'about' pictures. English for Specific Purposes, 41, 22-35.

Tapia-Ladino, M. \& Burdiles, G. (2009). Una caracterización del género informe escrito por estudiantes universitarios. Letras, 51(79), 17-49.

Tapia-Ladino, M., Burdiles, G. \& Arancibia, B. (2003). Aplicación de una pauta diseñada para evaluar informes académicos universitarios. Revista Signos, 36(54), 249-257.

Trigo, E. \& Núñez, X. (2018). Análisis competencial de la escritura académica en ELE de estudiantes portugueses. Aula de Encuentro, 20(2), 116-139. DOI:https://dx.doi. org/10.17561/ae.v20i2.7

Vásquez-Rocca, L. (2016). Estudio multidimensional del género Informe de Política Monetaria (IPOM) en el discurso de la economía (Tesis de doctoral). Pontificia Universidad Católica de Valparaíso.

Vásquez-Rocca, L. \& Parodi, G. (2015). Relaciones retóricas y multimodalidad en el género Informe de Política Monetaria del discurso académico de la Economía. Calidoscópio, 13(3), 388-405.

Venegas, R., Zamora, S. \& Galdames, A. (2016). Hacia un modelo retórico-discursivo del macrogénero Trabajo Final de Grado en Licenciatura. Revista Signos, 49(1), 247-279.

Walker, J. \& Sampson, V. (2013). Argument-driven inquiry: Using the laboratory to improve undergraduates' science writing skills through meaningful science writing, peerreview, and revision. Journal of Chemical Education, 90(10), 1269-1274.

Yeung, L. (2007). In search of commonalities: Some linguistic and rhetorical features of business reports as a genre. English for specific purposes, 26(2), 156-179. 